\title{
A seven-gene prognostic signature for rapid determination of head and neck squamous cell carcinoma survival
}

\author{
SIPENG SHEN ${ }^{1,2^{*}}$, JIANLING BAI ${ }^{1,2^{*}}$, YONGYUE WEI ${ }^{1,2}$, GUANRONG WANG $^{4}$, \\ QINGYA LI ${ }^{1,2}$, RUYANG ZHANG ${ }^{1,2}$, WEIWEI DUAN ${ }^{1,2}$, SHENG YANG $^{1,2}$, MULONG DU $^{1,2}$, \\ YANG ZHAO $^{1,2}$, DAVID C. CHRISTIANI ${ }^{2,5}$ and FENG CHEN ${ }^{1,2,3}$ \\ ${ }^{1}$ Department of Biostatistics, School of Public Health, ${ }^{2}$ China International Cooperation Center of \\ Environment and Human Health, ${ }^{3}$ Ministry of Education Key Laboratory for Modern Toxicology, School \\ of Public Health, Nanjing Medical University, Nanjing, Jiangsu; ${ }^{4}$ NPFPC Contraceptive Adverse Reaction \\ Surveillance Center, Jiangsu Institute of Planned Parenthood Research, Nanjing, Jiangsu, P.R. China; \\ ${ }^{4}$ Department of Environmental Health, Harvard School of Public Health, Boston, MA, USA
}

Received April 19, 2017; Accepted October 2, 2017

DOI: 10.3892/or.2017.6057

\begin{abstract}
Head and neck squamous cell carcinoma (HNSCC) is the sixth most common cancer and displays divergent clinical outcomes. Prognostic biomarkers might improve risk stratification and survival prediction. We aimed to investigate the prognostic genes associated with overall survival. A two-step gene selection method was used to develop a seven-genebased prognostic model based on the training set collected from The Cancer Genome Atlas (TCGA). In addition, the prognostic model was validated in an independent testing set from Gene Expression Omnibus (GEO). The score based on the model successfully distinguished HNSCC survival into high-risk and low-risk groups in the training set (HR, 2.79; 95\% CI, 1.98-3.92; $\left.\mathrm{P}=4.05 \times 10^{-9}\right)$ and the testing set (HR, 2.05; 95\% CI, 1.35-3.11; $\left.\mathrm{P}=7.98 \times 10^{-4}\right)$. In addition, the score could significantly predict 5 -year survival by ROC curves (AUCs for training set, 0.73 ; testing set, 0.66). Combining risk scores with clinical characteristics improved the AUCs beyond using clinical characteristics alone (training set, from 0.57 to 0.75 ; testing set, from 0.63 to 0.72 ). A subgroup sensitivity analysis with HPV status and tumor sites revealed that the risk score was significant in all subgroups except oral cavity tumors of the testing set. Furthermore, HPV-positive status improves survival in oropharyngeal HNSCC but not non-oropharyngeal HNSCC. In conclusion, the seven-gene prognostic signature
\end{abstract}

Correspondence to: Dr Feng Chen, Department of Biostatistics, School of Public Health, Nanjing Medical University, SPH Building, Room 412, 101 Longmian Avenue, Nanjing, Jiangsu 211136, P.R. China

E-mail: fengchen@njmu.edu.cn

${ }^{*}$ Contributed equally

Key words: head and neck squamous cell carcinoma, overall survival, gene expression, prognostic signature is a reliable and practical prognostic tool for HNSCC. This approach can add prognostic value to clinical characteristics and provides a new possibility for individualized treatment.

\section{Introduction}

Head and neck squamous cell carcinoma (HNSCC) constitutes approximately $4 \%$ of all new cancer diagnoses in the United States, with approximately 62,000 new cases in 2016 (1). Each year approximately 600,000 patients are affected worldwide (2). Importantly, the 5-year survival rate of HNSCC patients is only $40-50 \%$ (3). The high mortality rate is attributable to a high rate of late diagnosis, and the survival rate for cases in late stages is only $34.9 \%$ (4). These outcomes demonstrate the need for prognostic biomarkers to help predict patient outcome and outline individualized treatment plans. Age, clinical stage and smoking status are characteristics emerging as important contributors to clinical outcome that may also help us improve survival prediction (5-7). However, the traditional clinical information has limited prediction ability due to the complex molecular regulation mechanism in cancer.

Recently, the clinical importance of messenger RNA (mRNA) expression has been reported in various types of cancer including HNSCC. They play important roles in a variety of physiological and pathological processes, such as development, differentiation, cell proliferation, apoptosis and stress responses (8). Therefore, characterization of key genes in different tumors is essential not only for an urgent requirement of precision medicine (9) but also for preclinical and pharmaceutical research (10).

Prognostic models in HNSCC have been described using different biomarkers such as somatic mutations (11), microRNAs $(12,13)$ and proteins (4) but limited studies focus on mRNA expression according to Cancer Genetics Web (14). De Cecco et al (15) reported a gene expression survival predictor using HNSCC microarray data based on a semisupervised survival method involving principal component 
method (16). However, the model comprised 172 genes and was complicated for further interpretation. Now that transcriptome sequencing technologies (RNA-Seq) are being applied widely, there is a more ideal platform for cancer genetic studies (17). In addition, The Cancer Genome Atlas (TCGA) and Gene Expression Omnibus (GEO) repositories provide abundant HNSCC case resources, which may be useful to explore reliable biomarkers.

In this study, we investigated the prognostic value of seven gene expression biomarkers (AATF, APP, GNPDA1, HPRT1, $L A S P 1, P 4 H A 1$ and $I L F 3$ ) for HNSCC overall survival. The cases mainly included oropharyngeal, laryngeal and oral squamous cell carcinoma. TCGA cohort was used as the training set to generate the prognostic model by a two-stage variable selection. We also used an independent external testing set to validate the robustness and reproducibility of the model.

\section{Materials and methods}

Study population. Information for the HNSCC training set was obtained from TCGA on November 13, 2016 (18). Gene expression data were extracted from IlluminaHiSeq RNASeqV2 platform and normalized by RSEM method (19). In addition, we performed quality control with a total of 20530 genes. Genes with more than half of values as zero were removed, 17711 genes remained with quantile normalization. Patients with complete follow-up information and gene expression values for tumor tissues were included in the study. Information for the HNSCC testing set was collected from GSE65858 (20) in GEO. Gene expression data were extracted from Illumina HumanHT-12 V4.0 expression beadchip and normalized using the robust spline normalization (RSN) method (21). Consecutive patients with primary and metachronous secondary HNSCC of oral cavity, larynx, oro- and hypopharynx were included, while tumor cell lines and those with low quality assays were excluded. All gene expression values were $\log 2$-transformed and standardized for comparability between the training and testing sets.

Weighted t-test (WTT) method as the first step for gene selection. To select differentially expressed genes combined with clinical information, WTT was used to select genes based on the method of $\mathrm{Hu}$ et al (22). For the $i$ th subject with a covariate vector $Z_{i}$, the Cox proportional hazards model is given by $\lambda\left(t \mid Z_{i}\right)=\lambda_{0}(t) \exp \left(\beta^{T} Z_{i}\right)$ and the survival function is $S\left(t \mid Z_{i}\right)=\exp \left\{-\Lambda_{0}(t) \exp \left(\beta^{T} Z_{i}\right)\right\}$, where $\lambda_{0}(t)$ is the basic hazard function, $\beta$ is the regression coefficient and $\Lambda_{0}(t)$ is the cumulative baseline hazard function. Then we constructed a Cox regression model for each subject based on clinical information (age, sex, smoking status and clinical stage) only and defined $h_{i}=\beta^{T} Z_{i}$. The weights for $n$ patients totally were calculated accordingly:

$$
w_{i}=\frac{h_{i}}{\sum_{i=1}^{n} h_{i}} \times n
$$

which were assigned for the tumor cases but not the normal cases.

With the weighted tumor expression $\exp _{w i}=w_{i} \times \exp _{\mathrm{i}}$, Student's t-test was conducted for each gene to measure the difference between tumor and matched normal expression level. We also used $t$-test with no weight adjustment and examined the difference between the $t$-test statistics after and before weight adjustment, $d_{k}=t_{\text {adjust }}-t_{\text {unadjust }}$ for the $k$ th gene. Afterwards, 1,000 total permutations were performed and $d_{k i}$ could be got for the $i$ th permutation. Then, we calculated the averaged order statistics, $\bar{d}_{k}$, across all 1,000 permutations. A gene was labeled as significant when $\left|d_{k}-\bar{d}_{k}\right|$ was at the top $5 \%$.

Sure independence screening (SIS) as the second step for gene selection. After the WTT selection, there were still over 800 genes left, which were too many and not robust to build the prognostic sigature in HNSCC. The traditional univariate or multivariate Cox regression was not suitable to select the prognosis-associated genes because it easily led to overfitting and produced instable results (23). SIS was used to choose those which were truly associated with disease from the 5\% genes remaining for further modeling (24). This is a two-step screening approach: it first screened all genomic features and discarded the irrelevant features whose correlation with overall survival were weak, and secondly applied LASSO penalized regression to estimate the sensitivity from the selected genomic instability data. We could significantly reduce the number of genes in the final model by the SIS method.

Statistical analysis. Continuous variables are described as mean $\pm \mathrm{SD}$, and categorized variables are summarized by frequency (n) and proportion (\%). Chi-square test was used for rate or proportion comparison. Associations between the characteristics and the overall survival were evaluated by Cox proportional hazard models. Survival curves were drawn with the Kaplan-Meier method and were compared among subgroups using log-rank tests. To evaluate the robustness of the results, we used the bootstrap method with 'bootcov' function that computed a bootstrap estimate of the covariance matrix for a set of regression coefficients in $r m s$ package. The bootstrap procedure were carried out with 500 re-samplings for the multivariable Cox regression. We predicted 5-year patient survival using the nearest neighbor method for receiver operating characteristic (ROC) curves of censored survival data (25) and estimation of confidence intervals and P-values of area under the curve (AUC) was based on bootstrap resampling. In the subgroup analysis, we used the Fisher's exact test to compare the proportions of different HPV status or tumor sites.

Statistical analyses were performed using $\mathrm{R}$ version 3.3.1 (The R Foundation). P-values are two-sided and $\mathrm{P}<0.05$ indicates statistical significance.

\section{Results}

Demographic and clinical characteristics. The analysis included 512 HNSCC cases from TCGA training set and 270 cases from the GEO testing set (Table I). Cases in the training set had an average age of $60.8 \pm 11.9$ years, ranging from 19 to 90 years; 149 (29.1\%) individuals were followed until death. Cases in the testing set had an average age of $60.1 \pm 10.3$ years, ranging from 35 to 87 years; 88 (32.6\%) individuals were followed until death. 
Table I. Demographic and clinical characteristics of HNSCC patients.

\begin{tabular}{lcc}
\hline Characteristics & $\begin{array}{c}\text { Training set } \\
(\mathrm{n}=512)\end{array}$ & $\begin{array}{c}\text { Testing set } \\
(\mathrm{n}=270)\end{array}$ \\
\hline Median follow-up time (years) & 4.35 & 4.95 \\
Censor rate (\%) & 70.8 & 67.4 \\
Age, mean \pm SD (years) & $60.8 \pm 11.9$ & $60.1 \pm 10.3$ \\
Sex, n (\%) & & \\
Male & $376(73.4)$ & $223(82.6)$ \\
Female & $136(26.6)$ & $47(17.4)$
\end{tabular}

Smoking status, n (\%)

Never

Current/former

$\mathrm{NA}^{\mathrm{a}}$

115 (22.5)

48 (17.8)

$385(75.2) \quad 222(82.2)$

$12(2.3) \quad 0(0)$

Tumor site, n (\%)

Oropharynx ${ }^{\mathrm{b}}$

Larynx

Oral cavity ${ }^{\mathrm{b}}$

Others

$80(15.6) \quad 102(37.8)$

$114(22.3) \quad 48(17.8)$

$308(60.2) \quad 83(30.7)$

$10(2) \quad 37(13.7)$

HPV status, n (\%)

Positive

Negative

$\mathrm{NA}^{\mathrm{a}}$

T classification, $\mathrm{n}(\%)$

$\mathrm{T} 1$

T2

T3

T4

TX or $\mathrm{NA}^{\mathrm{a}}$

$\mathrm{N}$ classification, $\mathrm{n}(\%)$

N0

N1

$\mathrm{N} 2$

N3

$\mathrm{NX}$ or $\mathrm{NA}^{\mathrm{a}}$

$\mathrm{M}$ classification, $\mathrm{n}(\%)$

M0

M1

$\mathrm{MX}$ or $\mathrm{NA}^{\mathrm{a}}$

35 (6.8)

$241(47.1)$

$236(46.1)$

$73(27.0)$

$196(72.6)$

$1(0.4)$

48 (9.4)

$130(25.4)$

99 (19.3)

$172(33.6)$

$63(12.3)$

35 (13.0)

$80(29.6)$

58 (21.5)

97 (35.9)

$0(0)$

174 (34)

66 (12.9)

165 (32.2)

8 (1.6)

99 (19.3)

$484(94.5)$

$4(0.8)$

24 (4.7)

TNM stage, $\mathrm{n}(\%)$

I

II

III

IV

$\mathrm{NA}^{\mathrm{a}}$

20 (3.9)

97 (18.9)

$104(20.3)$

$278(54.3)$

13 (2.5)

61 (11.9)

$300(58.6)$

$122(23.8)$

7 (1.4)

$22(4.3)$
Table I. Continued.

\begin{tabular}{lcc}
\hline Characteristics & $\begin{array}{c}\text { Training set } \\
(\mathrm{n}=512)\end{array}$ & $\begin{array}{c}\text { Testing set } \\
(\mathrm{n}=270)\end{array}$ \\
\hline Neoadjuvant treatment, $\mathrm{n}(\%)$ & & \\
Yes & $10(1.9)$ & - \\
No & $502(98.1)$ & - \\
NA $^{\mathrm{a}}$ & $0(0)$ & $270(100)$ \\
\hline
\end{tabular}

${ }^{a} \mathrm{NA}$, not available; ${ }^{\mathrm{b}}$ oropharynx also includes tonsil and base of tongue; oral cavity also includes oral tongue, buccal mucosa, lip, alveolar ridge, hard palate and floor of mouth.

Development of biomarker signature model. To exclude a large number of genes unrelated to disease, we assessed the TCGA training set in two steps after quality control (Fig. 1A). First, of all 17711 genes with normalization, we used the WTT method to select the top $5 \%$ significant genes $(n=886)$ from 43 pairs of tumor and matched adjacent normal tissue data. All 886 genes were significantly differentially expressed (all $\mathrm{P} \leq 1.15 \times 10^{-5}$ ) and 199 genes were significant in univariate Cox regression analysis $(\mathrm{P}<0.05$; Fig. 1B). Second, the SIS method was used for further dimension reduction. After iterative process for different genes and LASSO penalized regression with 10-fold crossvalidation to select the best parameter, seven genes remained after selection. All of them were significantly overexpressed in tumor tissue (Fig. 1C). In addition, they were significantly associated with overall survival except LASP1 $(\mathrm{P}=0.056)$ (Fig. 1D). A Cox regression model was used to generate model coefficients. The biomarker signature model was calculated as risk score $=0.198 \mathrm{x} A A T F+0.244 \mathrm{x} A P P+0.252 \mathrm{x} G N P D A 1+$ $0.314 \mathrm{x} H P R T 1+0.136 \mathrm{x} L A S P 1+0.110 \mathrm{x} P 4 H A 1-0.388 \mathrm{x} I L F 3$. We categorized the patients into low-risk and high-risk groups and defined the cut-off value (score $=0.36$ ). This was selected by the optimum cut point according to the highest $\chi^{2}$ value defined by Kaplan-Meier survival analysis and log-rank test in the training test (26).

As a linear combination model of seven mRNAs, the risk score was significantly associated with the TCGA patient survival $\left(\mathrm{HR}_{\text {unadjust }}=2.79 ; 95 \% \mathrm{CI}, 1.98-3.92 ; \mathrm{P}=4.05 \times 10^{-9}\right)$ (Fig. 2A). In total, $16.4 \%$ in the low-risk group vs. $41.8 \%$ in the high-risk group were followed until death $\left(\chi^{2}=38.78\right.$, $\mathrm{P}=4.76 \times 10^{-10}$ ) (Fig. 2B). With the bootstrap adjustment for clinical characteristics, the results remained significant for all cases $\left(\mathrm{HR}_{\text {adjust }}=2.86 ; 95 \% \mathrm{CI}, 1.99-4.12 ; \mathrm{P}<0.0001\right)$ or cases with available HPV status $\left(\mathrm{HR}_{\text {adjust }}=3.17 ; 95 \% \mathrm{CI}\right.$, 1.90-5.30; $\mathrm{P}<0.0001$ ) (Table II).

Validation of the prognostic signature. In the GEO testing set, risk scores were calculated for each patient. Using the same cut-off value (score $=0.36$ ), the score showed a 2.05 times higher risk of death for the high-risk group compared to the low-risk group in univariate Cox regression $\left(\mathrm{HR}_{\text {unadjust }}=2.05\right.$; 95\% CI, 1.35-3.11; $\mathrm{P}=7.98 \times 10^{-4}$ ) (Fig. 2C). In total, $25.1 \%$ in the low-risk group vs. $46.3 \%$ in the high-risk group were followed until death $\left(\chi^{2}=11.62, \mathrm{P}=6.53 \times 10^{-4}\right)$ (Fig. 2D). Results retained statistical significance with further adjustment for covariates, 


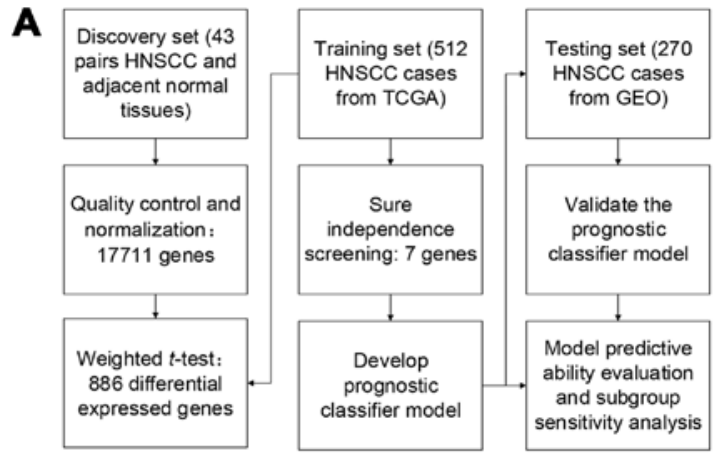

B

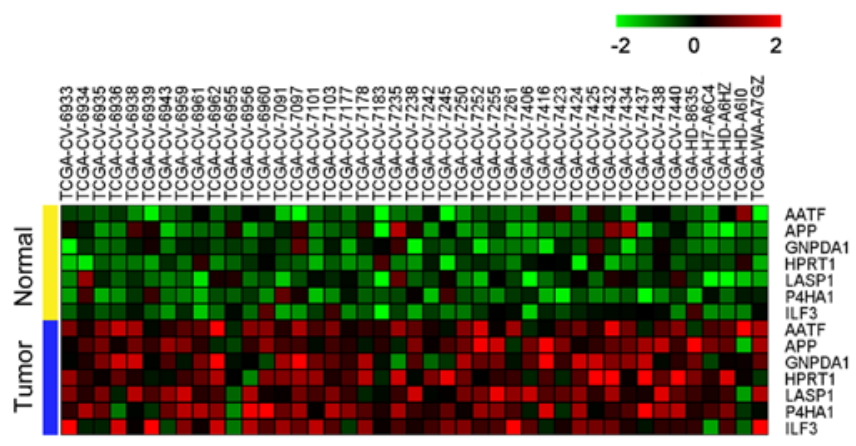

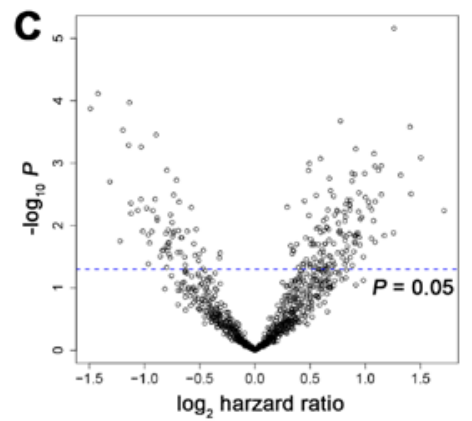

D

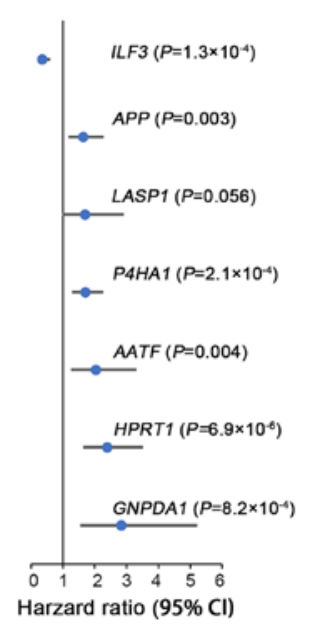

Figure 1. (A) Work flow of gene selection steps, with the number of genes remaining. (B) Heatmap of the seven genes using 43 pairs of tumor and matched adjacent normal HNSCC tissue data in the training set. The upper half is the expression of normal tissues, and the lower half is the expression of tumor tissues. (C) Volcano plot showing the prognostic significance of the 886 genes selected by the WTT method. This plot depicts the hazard ratio on the x-axis and statistical significance on the y-axis, as evaluated by the Cox regression model. The blue dashed line indicates a P-value of 0.05 . (D) Hazard ratio with $95 \% \mathrm{Cl}$ of the seven genes in univariable Cox regression analysis of the training set.

Table II. Multivariable Cox regression analysis of clinical characteristics and risk score.

\begin{tabular}{|c|c|c|c|c|c|c|}
\hline \multirow[b]{3}{*}{ Characteristics } & \multicolumn{4}{|c|}{ Training set } & & \\
\hline & \multicolumn{2}{|c|}{ All cases $(n=512)$} & \multicolumn{2}{|c|}{$\begin{array}{l}\text { Cases with HPV } \\
\text { status }(n=276)\end{array}$} & \multicolumn{2}{|c|}{ Testing set $(\mathrm{n}=270)$} \\
\hline & HR $(95 \% \mathrm{CI})$ & P-value & $\mathrm{HR}(95 \% \mathrm{CI})$ & P-value & $\mathrm{HR}(95 \% \mathrm{CI})$ & P-value \\
\hline High risk score & $2.86(1.99-4.12)$ & $<0.0001$ & $3.17(1.90-5.30)$ & $<0.0001$ & $1.94(1.27-2.96)$ & 0.002 \\
\hline Age (per year) & $1.02(1.00-1.04)$ & 0.039 & $1.01(0.99-1.04)$ & 0.141 & $1.03(1.00-1.06)$ & 0.020 \\
\hline Gender (Female) & $1.11(0.76-1.62)$ & 0.578 & $1.07(0.64-1.79)$ & 0.797 & $1.02(0.57-1.79)$ & 0.952 \\
\hline \multicolumn{7}{|l|}{ Smoking status } \\
\hline (Current/former smoker) & $1.26(0.80-1.98)$ & 0.324 & $1.56(0.87-2.79)$ & 0.133 & $0.92(0.49-1.75)$ & 0.815 \\
\hline Clinical stage (per stage) & $1.09(0.91-1.31)$ & 0.331 & $1.02(0.82-1.25)$ & 0.878 & $1.77(1.21-2.59)$ & 0.003 \\
\hline HPV status (positive) & - & - & $0.65(0.29-1.47)$ & 0.301 & $0.43(0.24-0.79)$ & 0.006 \\
\hline
\end{tabular}

Estimation of confidence intervals and P-values are based on a bootstrap estimate (500 resamples) of the variance-covariance matrix.

including age, sex, smoking status, HPV status and clinical stage $\left(\mathrm{HR}_{\text {adjust }}=1.94 ; 95 \% \mathrm{CI}, 1.27-2.96 ; \mathrm{P}=0.002\right)$ (Table II).

Furthermore, prognostic prediction ability for 5-year survival was evaluated. The time-dependent AUCs of risk scores for HNSCC cases were 0.73 (95\% CI, 0.68-0.78; $\mathrm{P}<0.001)$ in the training set (Fig. 3A) and 0.66 (95\% CI, 0.59-0.73;
$\mathrm{P}<0.001$ ) in the testing set (Fig. 3B). Besides, we combined the scores with clinical characteristics to see whether they could improve the predictive value. In the training set, prognostic score plus clinical characteristics had a higher AUC (AUC, 0.75; 95\% CI, 0.70-0.80) than the clinical characteristics (age, sex and stage) alone (AUC, 0.57; 95\% CI, 0.51-0.64) (Fig. 3C). 

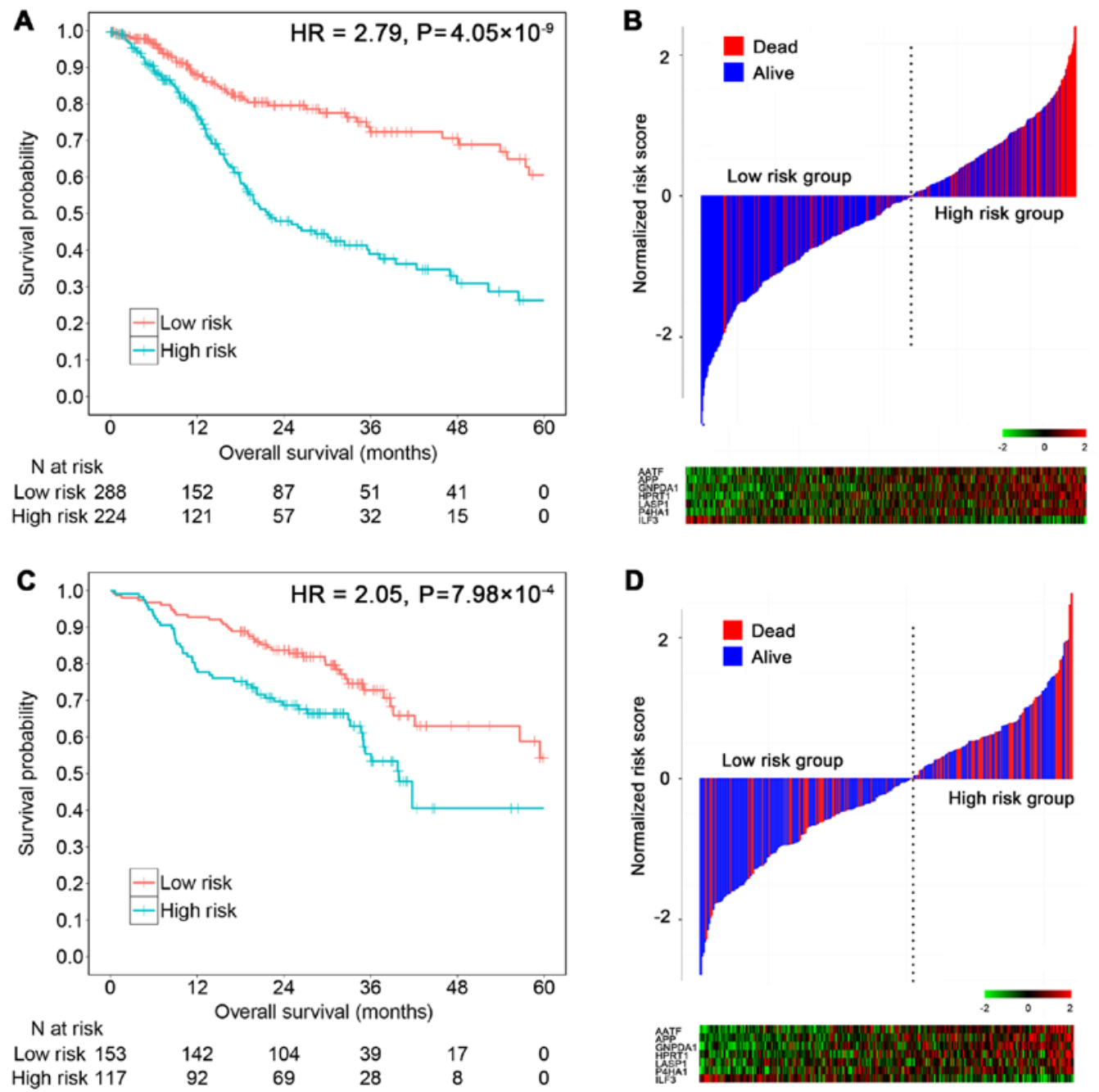

Figure 2. (A and B) Training set. (C and D) Testing set. Upper left panel: risk score distribution of the seven-gene model classifier and patient survival status. All scores are standardized ('score' $=$ score - 0.36) to make the low-risk group negative and high-risk group positive. Lower half panel: heatmap showing expression of the seven genes among tumor patients. Right panel: Kaplan-Meier analysis for the patients. The patients are divided into low-risk (red) and high-risk (blue) groups.

The testing set also displayed improvement in AUC from 0.63 (95\% CI, 0.57-0.70) to 0.72 (95\% CI, 0.65-0.78) (Fig. 3D). In brief, the risk score could better distinguish HNSCC prognosis beyond clinical information alone.

Subgroup sensitivity analysis with HPV status and tumor site. Next, we examined whether the risk score could help improve prognostication in the two datasets separately by subgroup sensitivity analysis. HPV-positive HNSCC has been widely recognized as associated with better prognosis than HPV-negative HNSCC (27). The risk score could significantly distinguish patient prognosis among 274 cases with available $\mathrm{HPV}$ information in the training set $\left(\mathrm{HPV}^{+}, \mathrm{P}=8.65 \times 10^{-6}\right.$; $\mathrm{HPV}^{-}, \mathrm{P}=1.24 \times 10^{-7}$; Fig. $4 \mathrm{~A}$ and $\left.\mathrm{B}\right)$ and 269 cases in the testing set regardless of $\mathrm{HPV}$ status $\left(\mathrm{HPV}^{+}, \mathrm{P}=0.004 ; \mathrm{HPV}^{-}, \mathrm{P}=0.014\right.$; Fig. 4C and D).

In different tumor sites, high vs. low-risk score significantly distinguished outcomes in patients with tumor at larynx (training set, $\mathrm{P}=0.001$; testing set, $\mathrm{P}=0.019$ ), oropharynx (training set, $\mathrm{P}=0.0004$; testing set, $\mathrm{P}=0.003$ ) and oral cavity in the training set $\left(\mathrm{P}=1.80 \times 10^{-5}\right)$ (Fig. 5). However, the result was not significant in oral cavity of the testing set $(\mathrm{P}=0.178)$, possibly due to the failure to distinguish patients with longer survival. In addition, we found that HPV-positive status improves survival in oropharyngeal HNSCC but not in nonoropharyngeal HNSCC (training set, $\mathrm{P}<0.001$; testing set, $\mathrm{P}=0.010$; Fig. $5 \mathrm{E}$ and $\mathrm{F}$ ), which was consistent with a previous report (28).

\section{Discussion}

In the present study, we developed an HNSCC prognostic risk model that includes seven mRNAs and validated it using an independent external data set. Integrating multiple biomarkers into an aggregated model could improve prognostic value compared with single biomarker (29). Results showed that the risk score was significantly associated with patient overall survival. HNSCC patients with higher risk scores tended to have a poorer clinical outcome. In addition, this score could improve model performance combined with clinical characteristics based on 5-year overall survival.

To screen out the survival-related genes from over 20,000 total genes, we used a two-stage screening method. The WTT method was used as the first step to identify a subset of genes 

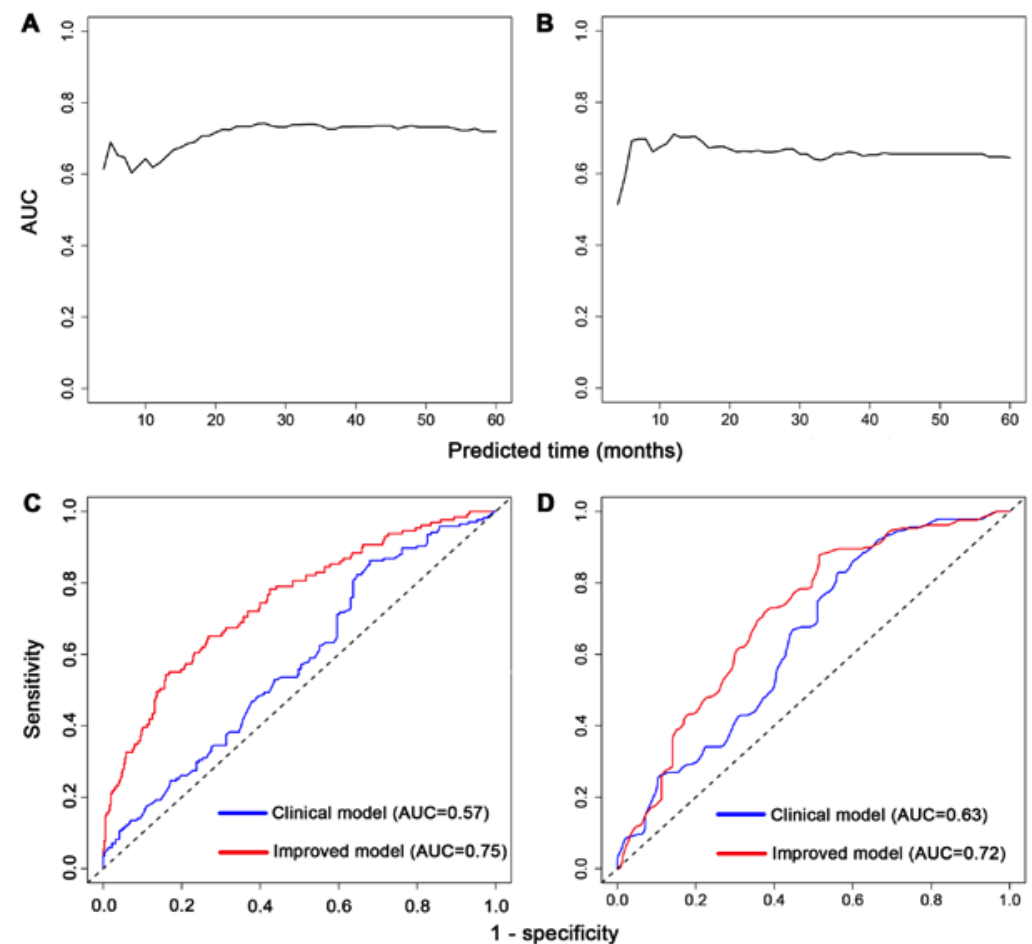

Figure 3. (A and B) Training set. (C and D) Testing set. Upper half panel: time-dependent ROC curves are used to evaluate patient survival with the risk score on different time, obtained by the nearest neighbor method. Lower half panel: ROC curves for clinical model and improved model. Clinical model contains age, sex, smoking status and clinical stage. Improved model contains the risk score and characteristics of clinical model.
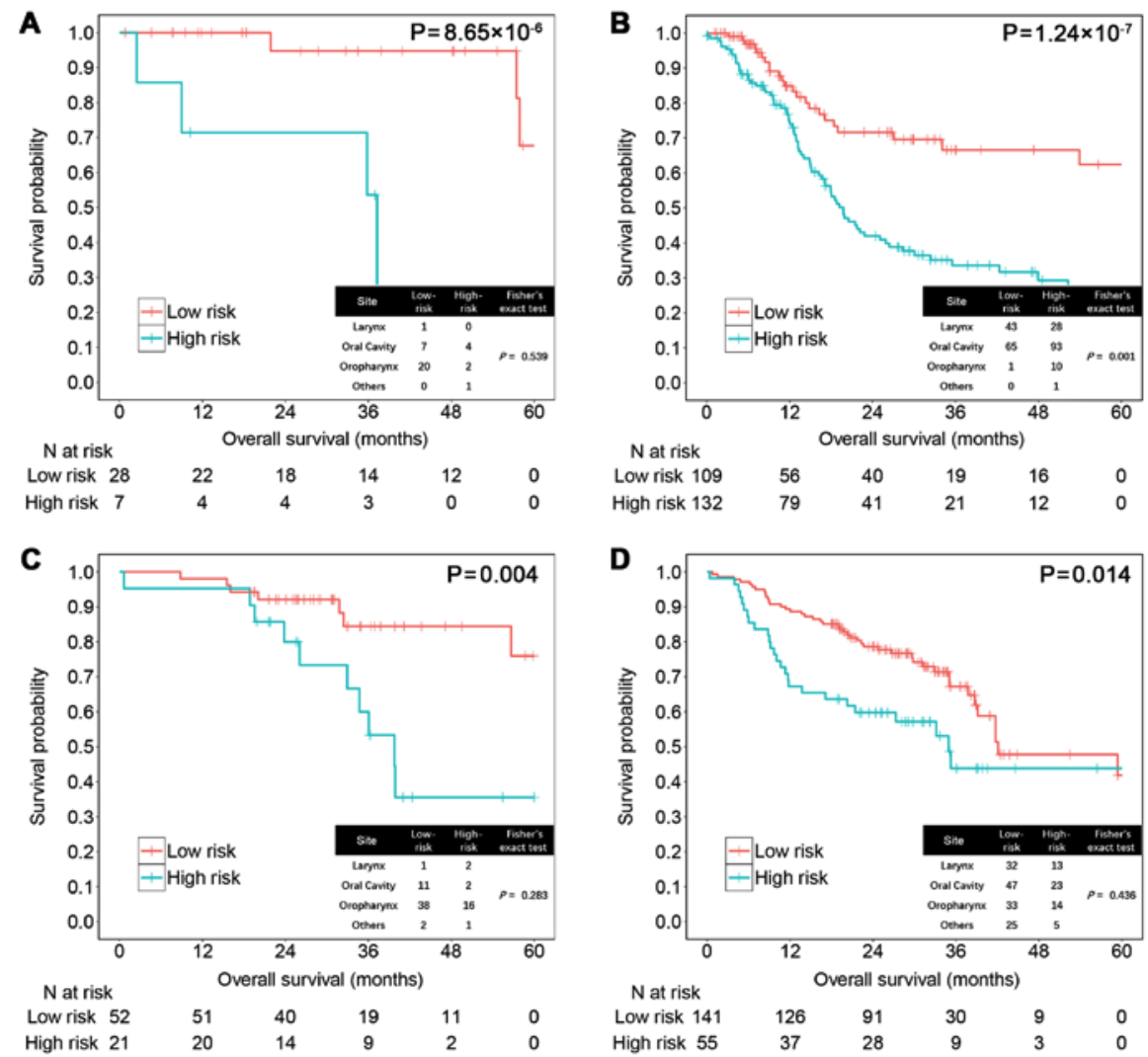

Figure 4. Subgroup sensitivity analysis with HPV status. (A) Training set $\left(\mathrm{HPV}^{+}\right)$. (B) Training set (HPV). (C) Testing set (HPV+). (D) Testing set (HPV). Cross tables of tumor sites and risk group proportions in each figure were summarized and tested by the Fisher's exact test.

that were not only differentially expressed in the tumor and matched normal tissues but also had an impact on patient survival by weighting the clinical covariates. Using permutation procedures that have widely been used in biomedical data 

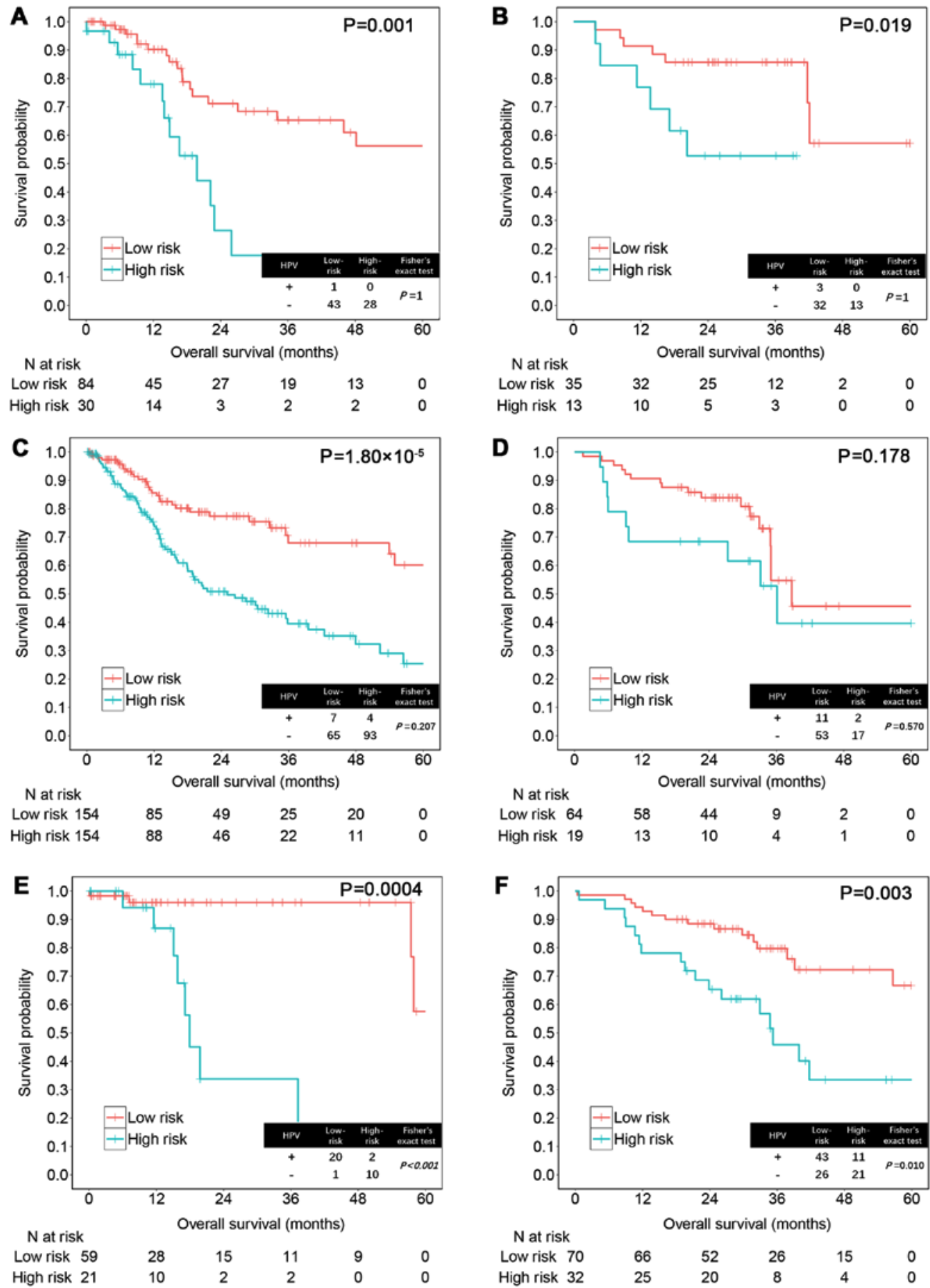

Figure 5. Subgroup sensitivity analysis with different tumor sites. (A and B) Laryngeal tumors. (C and D) Oral cavity tumors. (E and F) Oropharyngeal tumors. (A, C and E) Training set. (B, D and F) Testing set. Cross tables of HPV status and risk group proportions in each figure were summarized and tested by Fisher's exact test.

analysis (30), error rates can be controlled (22). The results show that all the selected genes were differentially expressed and number $(22.5 \%)$ were significantly associated with prognosis. The WTT method performs better than a traditional $t$-test, which will flag a large number of genes unrelated to disease. Afterwards, SIS was used to reduce the number of genes included in the final model as the second step. Compared with a traditional penalized regression like lasso or elastic net models, SIS is ungraded on the basis of penalized regression to reduce dimensionality from high to a moderate scale that is below the sample size (24). It has improved both speed and accuracy, and has a stronger association with disease (31). Of the seven genes in the training set, all of them are significantly differentially expressed in tumor and normal tissue. In addition, six of them are significant in a univariate Cox model and the last one shows suggestive significance $(\mathrm{P}=0.056)$. Our results show that the two-step gene selection method is amenable to deal with a high dimension problem.

Among the seven candidate genes, six have positive coefficients in the prognostic model and are associated with worse survival. AATF, also called Che-1, is a critical regulator of apoptosis driven by genes coding for PAR4 and p53 (32), 
and promotes tumor cell survival by sustaining mutant p53 transcription and inhibiting DNA damage response activation (33). It has activity in transcriptional regulation, cell cycle control, DNA damage responses, and in the execution of cell death programs (34). In addition, it interacts with NRAGE that has been found as a tumor marker in different cancers. AATF has been reported associated with multiple cancers, such as colon carcinoma (35), gastric cancer (36), hepatocellular carcinoma (37) and breast cancer (38).

$A P P$ was initially found to be associated with Alzheimer's disease, but it also contributes to regulating cell growth, apoptosis, and motility of cancer cells (39). Several studies have confirmed $A P P$ as an invaluable marker for oral carcinogenesis that promotes the proliferation and carcinogenesis of oral squamous cell carcinoma (OSCC) (40-42). Notably, in addition to its effects on promoting oral carcinogenesis, APP expression could be negatively regulated by tea in OSCC, which has been demonstrated to be effective in preventing animal carcinogenesis in different experimental systems (41).

$L A S P 1$, a recognized cancer biomarker functioning in cell structure, physiological processes, and cell signaling, contributes to cancer aggressiveness by overexpression (43). Increased LASPI levels occur in OSCC and more than ten other tumor types (44). It appears to involved in regulation of cancer cell metastatic propensity and perturb the architecture and dynamics of focal adhesion that triggers cell migration and invasion (45). In OSCC, LASPI plays an essential role in tumor cellular growth by mediating $\mathrm{G} 2 / \mathrm{M}$ transition.

This is not the first report for $P 4 H A l$ that was associated with HNSCC prognosis (46). It is involved in hydroxylation of collagen fibers and upregulated by HIFl under hypoxic conditions directly that drive a series of different biological processes related with malignant progression. $P 4 H A 1$ modulates target genes in cancer cell growth and tumor progression (47) and its expression increases during the invasion and metastasis of breast cancer and hepatoma as well $(48,49)$.

Furthermore, GNPDAl is an allosteric enzyme that catalyses the reversible conversion of D-glucosamine-6-phosphate into D-fructose-6-phosphate and ammonium (50). It has been reported upregulated in colorectal cancer cells with western blotting and immunofluorescence assay (51). The protein encoded by HPRT1 is a transferase, which plays a central role in the generation of purine nucleotides through the purine salvage pathway. It still needs further experiments to validate its prognostic value.

In contrast, ILF3 confers an onco-protective effect. Downregulation of $I L F 3$ can delay cell cycle progression, inhibit cell proliferation and reduce tumorigenic capacity in vivo (52). ILF3 is also involved in HPV-induced oncogenesis and p53-mediated apoptosis. It is a positive regulator of HPV E6 expression and its depletion leads to the accumulation of active p53 (53). Since HPV is effective in HNSCC, targeting on this gene may be useful to control the cancer.

The present study includes some limitations. First, in the subgroup analysis, the results may not strongly be robust due to small sample size of some groups. Second, due to the different experimental methods (RNASeq versus microarray) used between the two data sets, some bias may exist. Third, the differences between the two populations may indicate a need for further validation in another independent cohort for the current prognostic signature. Finally, the prognostic value of the seven genes in HNSCC still warrants further biological functional experiments.

In conclusion, our results showed that the seven-gene prognostic score significantly distinguishes HNSCC patients' prognosis and predicts 5-year overall survival in both training and testing sets. Thus, this score may be a novel biomarker based on gene expression levels and it warrants further investigation for establishing its relevance for clinical application.

\section{Acknowledgements}

We thank the patients and investigators who participated in TCGA and GEO for providing the data. The present study was supported by the National Natural Science Foundation of China (grant nos. 81530088 and 81473070 to F.C., 81302512 to J.B., 81402764 to Y.W. and 81402763 to R.Z.); and the Natural Science Foundation of Jiangsu, China (no. BK20140907 to Y.W.). It is also supported by the Nanjing Medical University international exchange and cooperation project (no. C018 to S.S.)

\section{References}

1. Siegel RL, Miller KD and Jemal A: Cancer statistics, 2016. CA Cancer J Clin 66: 7-30, 2016.

2. Cancer Genome Atlas N; Cancer Genome Atlas Network: Comprehensive genomic characterization of head and neck squamous cell carcinomas. Nature 517: 576-582, 2015.

3. Leemans CR, Braakhuis BJ and Brakenhoff RH: The molecular biology of head and neck cancer. Nat Rev Cancer 11: 9-22, 2011.

4. Chauhan SS, Kaur J, Kumar M, Matta A, Srivastava G, Alyass A, Assi J, Leong I, MacMillan C, Witterick I, et al: Prediction of recurrence-free survival using a protein expression-based risk classifier for head and neck cancer. Oncogenesis 4: e147, 2015.

5. Patel SG and Shah JP: TNM staging of cancers of the head and neck: Striving for uniformity among diversity. CA Cancer J Clin 55: 242-258, quiz 261-262, 264, 2005.

6. Wyss A, Hashibe M, Chuang SC, Lee YC, Zhang ZF, Yu GP, Winn DM, Wei Q, Talamini R, Szeszenia-Dabrowska N, et al: Cigarette, cigar, and pipe smoking and the risk of head and neck cancers: Pooled analysis in the International Head and Neck Cancer Epidemiology Consortium. Am J Epidemiol 178: 679-690, 2013.

7. Ang KK, Harris J, Wheeler R, Weber R, Rosenthal DI, Nguyen-Tân PF, Westra WH, Chung CH, Jordan RC, Lu C, et al: Human papillomavirus and survival of patients with oropharyngeal cancer. N Engl J Med 363: 24-35, 2010.

8. Kosaka N, Iguchi $\mathrm{H}$ and Ochiya T: Circulating microRNA in body fluid: A new potential biomarker for cancer diagnosis and prognosis. Cancer Sci 101: 2087-2092, 2010.

9. Longo DL: Tumor heterogeneity and personalized medicine. $\mathrm{N}$ Engl J Med 366: 956-957, 2012.

10. Zhao B, Hemann MT and Lauffenburger DA: Intratumor heterogeneity alters most effective drugs in designed combinations. Proc Natl Acad Sci USA 111: 10773-10778, 2014.

11. Mroz EA and Rocco JW: MATH, a novel measure of intratumor genetic heterogeneity, is high in poor-outcome classes of head and neck squamous cell carcinoma. Oral Oncol 49: 211-215, 2013.

12. Wong N, Khwaja SS, Baker CM, Gay HA, Thorstad WL, Daly MD, Lewis JS Jr and Wang X: Prognostic microRNA signatures derived from The Cancer Genome Atlas for head and neck squamous cell carcinomas. Cancer Med 5: 1619-1628, 2016.

13. Shi H, Chen J, Li Y, Li G, Zhong R, Du D, Meng R, Kong W and Lu M: Identification of a six microRNA signature as a novel potential prognostic biomarker in patients with head and neck squamous cell carcinoma. Oncotarget 7: 21579-21590, 2016.

14. SJ C: Home Page, Cancer Genetics Web: http://www.cancergenetics.org/index.htm Accessed: Feb 27, 2017.

15. De Cecco L, Bossi P, Locati L, Canevari S and Licitra L: Comprehensive gene expression meta-analysis of head and neck squamous cell carcinoma microarray data defines a robust survival predictor. Ann Oncol 25: 1628-1635, 2014. 
16. Bair E and Tibshirani R: Semi-supervised methods to predict patient survival from gene expression data. PLoS Biol 2: E108, 2004.

17. Giefing M, Wierzbicka M, Szyfter K, Brenner JC, Braakhuis BJ, Brakenhoff RH, Bradford CR, Sorensen JA, Rinaldo A, Rodrigo JP, et al: Moving towards personalised therapy in head and neck squamous cell carcinoma through analysis of next generation sequencing data. Eur J Cancer 55: 147-157, 2016.

18. Network TCGA: Comprehensive genomic characterization of head and neck squamous cell carcinomas. Nature 489: 519-525, 2012.

19. Li B and Dewey CN: RSEM: Accurate transcript quantification from RNA-Seq data with or without a reference genome. BMC Bioinformatics 12: 323, 2011

20. Wichmann G, Rosolowski M, Krohn K, Kreuz M, Boehm A, Reiche A, Scharrer U, Halama D, Bertolini J, Bauer U, et al; Leipzig Head and Neck Group (LHNG): The role of HPV RNA transcription, immune response-related gene expression and disruptive TP53 mutations in diagnostic and prognostic profiling of head and neck cancer. Int J Cancer 137: 2846-2857, 2015.

21. Shi W, Oshlack A and Smyth GK: Optimizing the noise versus bias trade-off for Illumina whole genome expression BeadChips. Nucleic Acids Res 38: e204-e204, 2010.

22. Hu J, Yin G, Morris JS, Zhang L and Wright FA: Entropy and survival-based weights to combine affymetrix array types and analyze differential expression and survival. In: Methods of Microarray Data Analysis. Shoemaker JS and Lin SM (eds) CAMDA Springer, Boston, MA, pp95-108, 2004.

23. van Houwelingen HC, Bruinsma T, Hart AA, Van't Veer LJ and Wessels LF: Cross-validated Cox regression on microarray gene expression data. Stat Med 25: 3201-3216, 2006.

24. Fan J and Lv J: Sure independence screening for ultrahigh dimensional feature space. J R Stat Soc Ser A Stat Soc 70: 883-911, 2008.

25. Heagerty PJ, Lumley T and Pepe MS: Time-dependent ROC curves for censored survival data and a diagnostic marker. Biometrics 56: 337-344, 2000

26. Wei JH, Haddad A, Wu KJ, Zhao HW, Kapur P, Zhang ZL, Zhao LY, Chen ZH, Zhou YY,Zhou JC, et al: A CpG-methylationbased assay to predict survival in clear cell renal cell carcinoma. Nat Commun 6: 8699, 2015.

27. Mroz EA, Tward AD, Hammon RJ, Ren Y and Rocco JW: Intratumor genetic heterogeneity and mortality in head and neck cancer: Analysis of data from the Cancer Genome Atlas. PLoS Med 12: e1001786, 2015.

28. Worsham MJ, Stephen JK, Chen KM, Mahan M, Schweitzer V, Havard S and Divine G: Improved survival with HPV among African Americans with oropharyngeal cancer. Clin Cancer Res 19: 2486-2492, 2013.

29. Ng SW, Mitchell A, Kennedy JA, Chen WC, McLeod J, Ibrahimova N, Arruda A, Popescu A, Gupta V, Schimmer AD, et al: A 17-gene stemness score for rapid determination of risk in acute leukaemia. Nature 540: 433-437, 2016.

30. Ludbrook J and Dudley H: Why permutation tests are superior to $\mathrm{t}$ and $\mathrm{F}$ tests in biomedical research. Am Stat 52: 127-132, 1998.

31. Fan J and Lv J: Sure independence screening for ultra-high dimensional feature space (with discussion). J R Stat Soc Ser A Stat Soc 70: 849-911, 2008.

32. Sharma S, Kaul D, Arora M and Malik D: Oncogenic nature of a novel mutant AATF and its interactome existing within human cancer cells. Cell Biol Int 39: 326-333, 2015.

33. Bruno T, Desantis A, Bossi G, Di Agostino S, Sorino C, De Nicola F, Iezzi S, Franchitto A, Benassi B, Galanti S, et al: Che-1 promotes tumor cell survival by sustaining mutant p53 transcription and inhibiting DNA damage response activation. Cancer Cell 18: 122-134, 2010

34. Passananti $\mathrm{C}$ and Fanciulli M: The anti-apoptotic factor Che-1/ AATF links transcriptional regulation, cell cycle control, and DNA damage response. Cell Div 2: 21, 2007.

35. Di Padova M, Bruno T, De Nicola F, Iezzi S, D'Angelo C, Gallo R, Nicosia D, Corbi N, Biroccio A, Floridi A, et al: Che-1 arrests human colon carcinoma cell proliferation by displacing HDAC1 from the $\mathrm{p} 21^{\mathrm{WAF} 1 / \mathrm{CIP} 1}$ promoter. J Biol Chem 278: 36496-36504, 2003.
36. Kanda M, Shimizu D, Fujii T, Tanaka H, Tanaka Y, Ezaka K, Shibata M, Takami H, Hashimoto R, Sueoka S, et al: Neurotrophin receptor-interacting melanoma antigen-encoding gene homolog is associated with malignant phenotype of gastric cancer. Ann Surg Oncol 23 (Suppl 4): 532-539, 2016.

37. Shimizu D, Kanda M, Sugimoto H, Sueoka S, Takami H, Ezaka K, Tanaka Y, Hashimoto R, Okamura Y, Iwata N, et al: NRAGE promotes the malignant phenotype of hepatocellular carcinoma. Oncol Lett 11: 1847-1854, 2016.

38. Sharma M: Apoptosis-antagonizing transcription factor (AATF) gene silencing: Role in induction of apoptosis and down-regulation of estrogen receptor in breast cancer cells. Biotechnol Lett 35: 1561-1570, 2013

39. Lim S, Yoo BK, Kim HS, Gilmore HL, Lee Y, Lee HP, Kim SJ, Letterio J and Lee HG: Amyloid- $\beta$ precursor protein promotes cell proliferation and motility of advanced breast cancer. BMC Cancer 14: 928, 2014. https://doi.org/10.1186/1471-2407-14-928.

40. Provenzano MJ, Yu L, Hitchler MJ, Fitzgerald MP, Robinson RA, Wayne S, Ver Meer M and Domann FE: AP-2 participates in the transcriptional control of the amyloid precursor protein (APP) gene in oral squamous cell carcinoma. Exp Mol Pathol 83: 277-282, 2007.

41. Ko SY, Chang KW, Lin SC, Hsu HC and Liu TY: The repressive effect of green tea ingredients on amyloid precursor protein (APP) expression in oral carcinoma cells in vitro and in vivo. Cancer Lett 245: 81-89, 2007.

42. Ko SY, Lin SC, Chang KW, Wong YK, Liu CJ, Chi CW and Liu TY: Increased expression of amyloid precursor protein in oral squamous cell carcinoma. Int J Cancer 111: 727-732, 2004.

43. Orth MF, Cazes A, Butt E and Grunewald TG: An update on the LIM and SH3 domain protein 1 (LASP1): A versatile structural, signaling, and biomarker protein. Oncotarget 6: 26-42, 2015.

44. Shimizu F, Shiiba M, Ogawara K, Kimura R, Minakawa Y, Baba T, Yokota S, Nakashima D, Higo M, Kasamatsu A, et al: Overexpression of LIM and SH3 protein 1 leading to accelerated $\mathrm{G} 2 / \mathrm{M}$ phase transition contributes to enhanced tumourigenesis in oral cancer. PLoS One 8: e83187-e83187, 2013.

45. Ruggieri V, Agriesti F, Tataranni T, Perris R and Mangieri D: Paving the path for invasion: The polyedric role of LASP1 in cancer. Tumour Biol 39: 1010428317705757, 2017.

46. Tawk B, Schwager C, Deffaa O, Dyckhoff G, Warta R, Linge A Krause M, Weichert W, Baumann M, Herold-Mende C, et al: Comparative analysis of transcriptomics based hypoxia signatures in head- and neck squamous cell carcinoma. Radiother Oncol 118: 350-358, 2016

47. Chakravarthi BVSK, Pathi SS, Goswami MT, Cieślik M, Zheng H, Nallasivam S, Arekapudi SR, Jing X, Siddiqui J, Athanikar J, et al: The miR-124-prolyl hydroxylase P4HA1MMP1 axis plays a critical role in prostate cancer progression. Oncotarget 5: 6654-6669, 2014

48. Feng G, Shi H, Li J, Yang Z, Fang R, Ye L, Zhang W and Zhang X: MiR-30e suppresses proliferation of hepatoma cells via targeting prolyl 4-hydroxylase subunit alpha-1 (P4HA1) mRNA. Biochem Biophys Res Commun 472: 516-522, 2016.

49. Gilkes DM, Chaturvedi P, Bajpai S, Wong CC, Wei H, Pitcairn S, Hubbi ME, Wirtz D and Semenza GL: Collagen prolyl hydroxylases are essential for breast cancer metastasis. Cancer Res 73: 3285-3296, 2013.

50. Arreola R, Valderrama B, Morante ML and Horjales E: Two mammalian glucosamine-6-phosphate deaminases: A structural and genetic study. FEBS Lett 551: 63-70, 2003

51. He YJ, Li WL, Liu BH, Dong H, Mou ZR and Wu YZ: Identification of differential proteins in colorectal cancer cells treated with caffeic acid phenethyl ester. World J Gastroenterol 20: 11840-11849, 2014.

52. Jiang W, Huang H, Ding L, Zhu P, Saiyin H, Ji G, Zuo J, Han D, Pan Y, Ding D, et al: Regulation of cell cycle of hepatocellular carcinoma by NF90 through modulation of cyclin E1 mRNA stability. Oncogene 34: 4460-4470, 2015.

53. Shamanna RA, Hoque M, Pe'ery T and Mathews MB: Induction of p53, p21 and apoptosis by silencing the NF90/NF45 complex in human papilloma virus-transformed cervical carcinoma cells. Oncogene 32: 5176-5185, 2013. 